\title{
SAZONALIDADES DO IBOVESPA
}

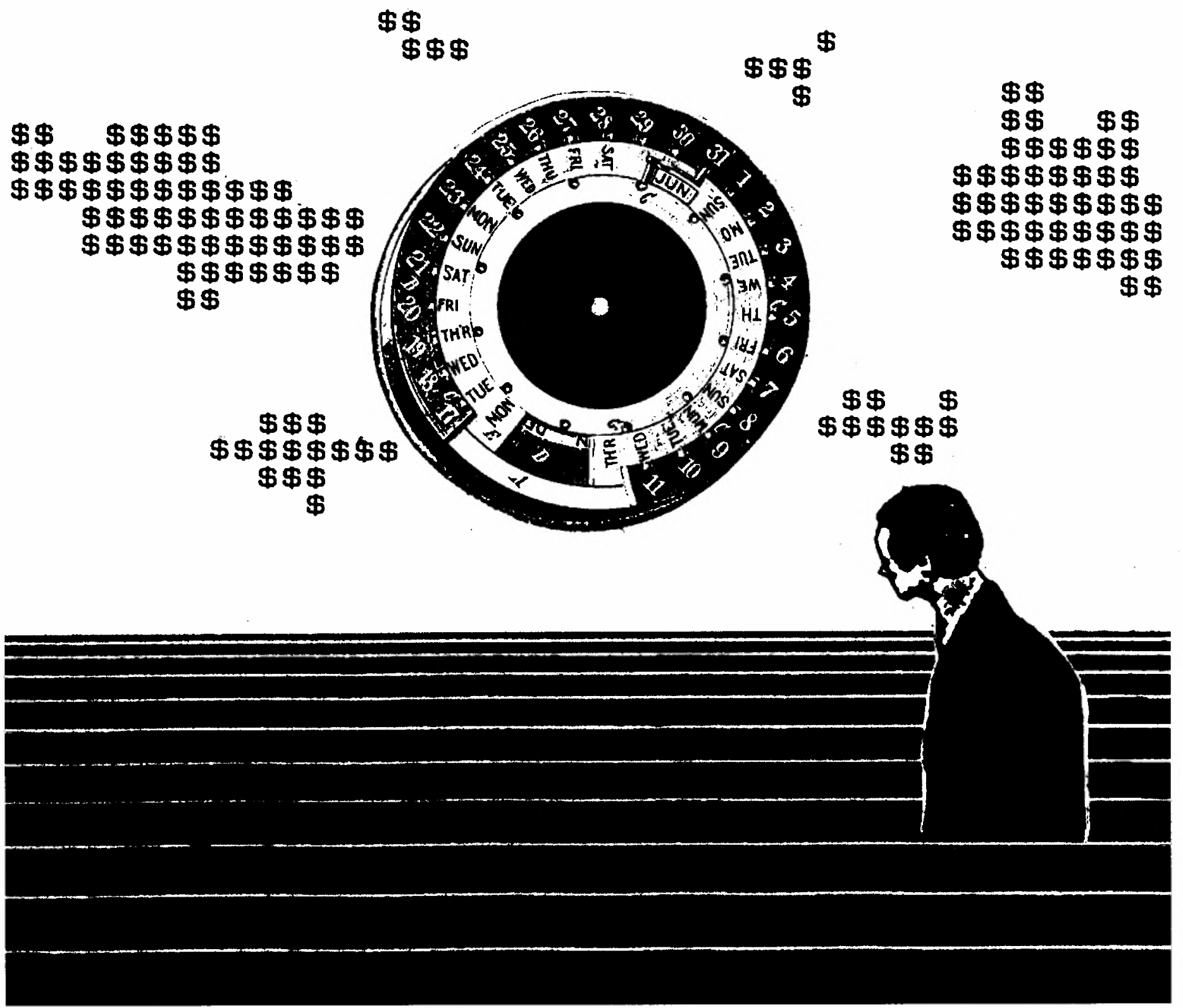

Newton C. A. da Costa Jr.

Doutorando da EAESP/FGV e "Research

Student" da Universidade de Lancaster.

* RESUMO: Neste trabalho são examinadas as sazonalidades dia-da-semana e mês-do-ano nos retornos do IBOVESPA através de métodos estatísticos paramétricos e não-paramétricos. A sazonalidade dia-da-semana foi observada e é semelhante à do mercado de capitais americano, onde o menor retorno é na segunda-feira e o maior na sexta-feira.
* PALAVRAS-CHAVES: Mercado eficiente, sazonalidades, anomalias, efeito mês-do-ano, efeito dia-da-semana.

* ABSTRACT: This study examines the day-of-theweek and the month-of-the-year effects in the São Paulo Stock Exchange Index (IBOVESPA). The observed dayof-the-week effect was similar to the U.S. stock market, showing low returns on Mondays and high returns on Fridays.

* KEY WORDS: Efficient market, seasonableness, anomalies, month-of-the-year effect, day-of-the-week effect. 


\section{INTRODUÇĀO}

$\mathrm{E}$ $\mathrm{m}$ Finanças há dois paradigmas principais e a maior parte das pesquisas na área ou os tem como supostos ou tenta verificar sua validade.

O primeiro é a hipótese do mercado eficiente, que segundo E. F. Fama ${ }^{1}$ é o mercado onde os preços refletem totalmente as informações disponíveis. Esta definição pressupõe os seguintes postulados:

- não existência de custos de transação;

- todas as informações estão disponíveis para todos os investidores a um custo zero;

- os investidores têm expectativas homogêneas.

Assim, um mercado é considerado eficiente se a posse de um conjunto de informações $\left(I_{t}\right)$ sobre esse mercado não alterar o retorno esperado em investir no mesmo. Ou seja, $\mathbf{E}\left(\mathbf{R}_{\mathrm{t}+1} / \mathbf{I}_{\mathrm{t}}\right)=\mathrm{E}\left(\mathbf{R}_{\mathrm{t}}\right)$.

No entanto, a definição acima é difícil de ser testada na prática. M.C.Jensen ${ }^{2}$ fornece outra definição que se tornou a base de muitos estudos na área: " $u m$ mercado é eficiente em relação a determinado conjunto de informações, $\left(\mathrm{I}_{\mathrm{t}}\right)$, se for impossivel obter qualquer lucro econômico com base em $\left(\mathrm{I}_{\mathrm{t}}\right)$. Por lucro econômico entendese o retorno ajustado ao risco, descontando-se todos os custos". Assim, se existirem estratégias de investimento que, descontando-se todos os custos, ainda proporcionem lucro, então o mercado não será considerado eficiente.

O segundo paradigma é o modelo de precificação de ativos de capital (CAPM), que é um modelo teórico que fornece uma maneira de se determinar o valor de um ativo de risco, dada a função de probabilidade conjunta de seus valores futuros. Foi inicialmente formulado por W.F.Sharpe ${ }^{3}$ e J.Lintner ${ }^{4}$ e ampliado por R.C.Merton 5 .

Em sua versão mais simplificada, o CAPM pode ser formalizado pela seguinte equação:

$$
E\left(R_{i}\right)=r_{f}+B_{i}\left[E\left(R_{m}\right)-r_{f}\right]
$$

onde:

$\mathbf{R}_{\mathbf{i}}$ é o retorno esperado para o ativo i;

$\mathbf{r}_{\mathrm{f}}$ é o retorno livre de risco; do;

$\mathbf{E}\left(\mathbf{R}_{\mathbf{m}}\right)$ é o retorno esperado do portfólio de merca-

$B_{\mathbf{i}}$ é uma medida do risco do ativo i em relação ao risco do mercado, dado por $\operatorname{cov}\left(\mathbf{R}_{\mathbf{i}}, \mathbf{R}_{\mathrm{m}}\right) / \operatorname{var}\left(\mathbf{R}_{\mathrm{m}}\right)$.

Dessa maneira, se um mercado se comporta de acordo com o CAPM, o valor esperado dos ativos de risco será igual ao retorno do ativo livre de risco mais um prêmio pelo risco incorrido em se investir no ativo em apreço.

Durante as décadas de 60 e 70 , muitas publicações em teoria financeira tentavam comprovar os dois paradigmas acima mencionados. $\mathrm{E}$ a conclusão a que se chegava era que o mercado se comportava de uma maneira eficiente.

Com o desenvolvimento de computadores cada vez mais poderosos e de banco de dados cada vez maiores e mais completos, grande parte das pesquisas começou a detectar certas anomalias quanto ao comportamento dos retornos de ativos financeiros que antes não haviam sido notadas, anomalias que iam de encontro com as hipóteses de eficiência de mercado. A maior parte desses estudos surgiu nos Estados Unidos, com testes em ações ordinárias. Posteriormente, resultados semelhantes foram encontrados em outros tipos de mercados e em outros países.

Entre as principais anomalias detectadas nesses estudos, têm-se: efeito dia-da-semana, efeito mês-doano, efeito tamanho-da-firma, efeito preço/lucro.

\section{Efeito Dia-da-Semana}

O efeito dia-da-semana diz respeito ao fato de os retornos diários de ativos de risco serem diferentes ao longo dos dias da semana. A maioria das pesquisas a esse respeito detectou um retorno menor nos primeiros dias da semana quando comparados aos últimos dias. Isso aparentemente contraria a hipótese do mercado eficiente. Essa anomalia foi detectada em diversos estudos tais como Cross, French, Gibbons e Hess, Lakonishok e Levi, Rogalski, Keim e Stambaugh ${ }^{6}$ etc., no mercado americano. E Santesmases, Condoyanni, O'Hanlon e Ward, Ball e Bowers, Theobald e Price ${ }^{7}$ etc., em mercados de outros países.

1. FAMA, E.F. "Efficient Capital Markets: A Review of Theory and Empirical Word". Journal of Finance, 25:383-417, 1970.

2. JENSEN, M.C. "Some Anomalous Evidence Regarding Market Efficiency". Journal of Financial Economics, 6:95-101, 1978.

3. SHARPE, W.F. "Capital Asset Prices: A Theory of Market Equilibrium under Conditions of Risk". Journal of Finance, 19.425-442, 1964.

4. LINTNER, J. "The Valuation of Risk Assets and the Selection of Risk Investments in Stock Portfolios and Capital Budgets". Review of Economics and Statistics, 47:13-37, 1965.

5. MERTON, R.C. "An Intertemporal Capital Asset Pricing Model". Econometrica, setembro, 1973, pp. 867-887.

6. CROSS, F. "The Behaviour of Stock Prices on Fridays and Mondays". Financial Analysts Journal, novembro/ dezembro, 1973, pp. 67-69; FRENCH, K.R. "Stock Returns and the Weekend Effect". Journal of Financial Economics, 8:55-69, 1980; GIBBONS, M. \& HESS, P. "Day of the Week Effects and Asset Returns". Journal of Business, 54:579-596, 1981; LAKONISHOK, J. \& LEVI, M. "Weekend Effects on Stock Returns: A Note". Journal of Finance, 37:883-889, 1982; ROGALSKI, R. "New Findings Regarding Day of the Week Returns over Trading and Non-Trading Periods: A Note". Journal of Finance, 39:1603-1614, 1984; KEIM, D.B. \& STAMBAUGH, R.F. "A Further Investigation of the Weekend Effect in Stock Returns". Journal of Finance, 39.819-839, 1984.

7. SANTESMASES, M. "An Investigation of the Spanish Stock Market Seasonalities". Journal of Business Finance and Accounting, 13:267276, 1986; CONDOYANNI, L.J. et alii. "Day of the Week Effects on 


\section{Efeito mês-do-Ano}

$\mathrm{O}$ efeito mês-do-ano é devido à existência de retornos mensais de ativos de risco diferentes, ao longo dos meses do ano. Também conhecido por efeito janeiro, em virtude de as primeiras pesquisas terem sido feitas no mercado americano e de lá ter sido detectado um retorno no mês de janeiro maior que o dos outros meses do ano. Wachtel ${ }^{8}$ foi o primeiro a observar esse fenômeno, porém foram Rozeff e Kinney ${ }^{9}$ os primeiros a estudar essa anomalia com maior detalhe. Outros estudos no mercado americano foram feitos por Tinic e West, Keim, Reinganum, Banz, Brown, Kleidom e Marsh ${ }^{10}$ etc. Fora dos Estados Unidos, podem-se citar os trabalhos de Gultekin e Gultekin, Kato e Schallheim, Brown, Keim, Kleidon e Marsh, Santesmases, Levis ${ }^{11}$ entre outros.

\section{Efeito Tamanho-da-Firma}

O efeito tamanho-da-firma diz respeito aos retornos ajustados ao risco das ações das pequenas empresas serem maiores do que os retornos ajustados ao risco das ações de empresas com maior capitalização. $\mathrm{O}$ primeiro autor a detectar essa anomalia foi Banz ${ }^{12}$. Também têm-se os trabalhos de Roll, Reinganum, Brown, Kleidon e Marsh, Keim ${ }^{13}$, entre outros.

\section{Efeito Preço-Lucro}

O efeito preço/lucro está em que, usando-se uma estratégia de investir em ações com baixo índice preço/lucro, se obtém um retorno ajustado ao risco acima do previsto pelos diversos modelos de CAPM conhecidos. O trabalho mais conhecido a esse respeito é o de Basu ${ }^{14}$. Outros trabalhos são os de Cook e Rozeff, Reinganum, Keim ${ }^{15}$ etc.

Neste trabalho, usando-se cotações mensais do IBOVESPA durante os últimos 20 anos e cotações diárias durante um período de 39 meses, aplicam-se algumas das metodologias das pesquisas acima citadas para determinar se os efeitos dia-da-semana e mês-do-ano também são encontradas no mercado brasileiro.

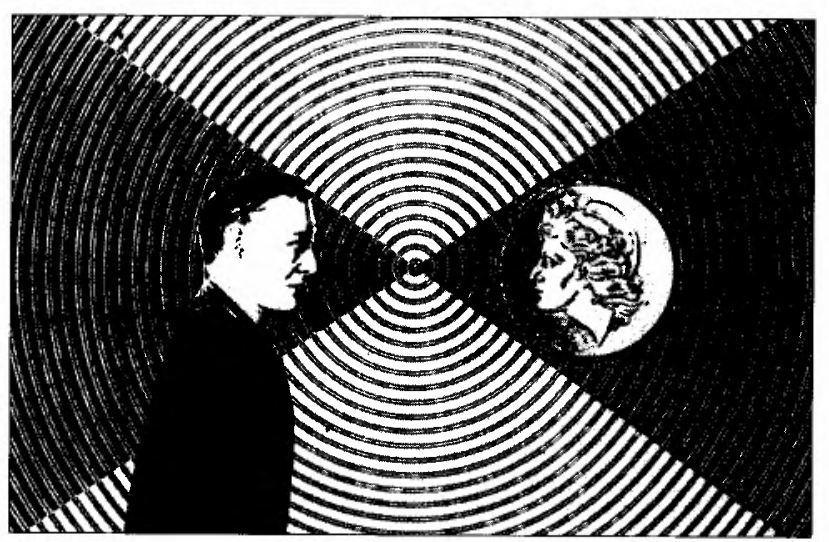

\section{O EFEITO MÉS-DO-ANO}

Para detectar o efeito mês-do-ano, foram analisadas as cotações mensais do IBOVESPA durante o período de 1969 a 1988. Foram analisadas tanto a série dos retornos não deflacionada como a deflacionada. No deflacionamento da série, foi usado o Índice Geral de Preços-disponibilidade interna (IGP-di).

Para o cálculo dos retornos mensais, foi suposto

Stock Returns: International Evidence". Journal of Business Finance and Accounting, 14.159-174, 1987; BALL, R. \& BOWERS, J. “Daily Seasonals in Equity and Fixed-Interest Returns: Australian Evidence and Test of Plausible Hypothesis". In: DIMSON, E. (org.) Stock Market Anomalies. Cambridge University Press, 1988; THEOBALD, M. \& PRICE, V. "Seasonality Estimation in Thin Markets". Journal of Finance, 39.377-392, 1984.

8. WACHTEL, S. "Certain Observations on Seasonal Movements in Stock Prices". Journal of Business, 15:184-193, 1946.

9. ROZEFF, M. \& KINNEY. W. "Capital Market Seasonality: The Case of Stock Market Returns". Journal of Financial Economics, 2:379-402, 1976.

10. TINIC, S.M. \& WEST, R.R. "Risk and Return". Journal of Financial Economics, 13:561-574, 1984; KEIM, D.B. "Size-Related Anomalies and Stock Return Seasonality: Further Empirical Evidence". Journal of Financial Economics, 12.13-32, 1983; REIGANUM, M.R. "The Anomalous Stock Market Behaviour Return of Small Firms in January: Empirical Tests for Tax-Loss Selling Effects". Journal of Financial Economics, 12.89-104, 1983; BANZ R.W. "The Relationship Between Return and Market Value of Common Stock". Journal of Financial Economics, 9.3-18, 1981; BROWN, P. et alii. "New Evidence on the Nature of Size-Related Anomalies in Stock Prices". Journal of Financial Economics, 12.33-56, 1983.

11. GULTEKIN, M.N. \& GULTEKIN, N.B. "Stock Market Seasonality: International Evidence". Journal of Financial Economics, 12:469-481, dezembro, 1983; KATO, K. \& SCHALLHEIM, S. "Seasonal and Size Anomalies in the Japanese Stock Market". Journal of Financial and Quantitative Analysis, 20.243-260, fevereiro, 1985; BROWN, P. et alii. "Stock Return Seasonalities and the Tax-Loss Selling Hypothesis: Analysis of the Arguments and Australian Evidence". Journal of Financial Economics, 12:105-127, junho, 1983; SANTESMASES, M. Op. cit.; LEVIS, M. "Are Small Firms Big Performers". The Investment Analyst, 76: 21-26, abril, 1985.

12. BANZ, R.W. Op. cit.

13. ROLL, R. "A Possible Explanation of the Small Firm Effect", Journal of Finance, 36:879-888, setembro, 1981; REINGANUM, M.R. "Misspecification of Capital Asset Pricing: Empirical Anomalies Based on Earnings'Yields and Market Values". Journal of Financial Economics, 9:19-46, 1981; __. .A Direct Test of Roll's Conjecture on the Firm Size Effect". Journal of Finance, 37: 27-35, 1982; BROWN, P. et alii. "New Evidence on the Nature of Size-Related Anomalies in Stock Prices". Op. cit.; KEIM, D.B. Op. cit.

14. BASU, S. "Investment Performance of common Stocks in Relation to their Price/Earnings Ratios: A Test of the Efficient Market Hypothesis". Journal of Finance, junho, 1977, pp. 663-682;

"The Relationship Between Earnings Yelds, Market Value and the Returns for NYSE Stocks: Further Evidence". Journal of Financial Economics, junho, 1983, pp. 129-156.

15. COOK, T.J. \& ROZEFF, M.S. "Size and Earnings/Price Ratio Anomalies: One Effect or Two?". Journal of Financial and Quantitative Analysis, 13:449-466, 1984; REINGANUM, M.R. "Misspecification of Capital Asset Pricing: Empirical Anomalies Based on Earnings'Yields and Market Values". Op. cit.; KEIM, D.B. "Stock Market Regularities: A Synthesis of the Evidence and Explanations". In: DIMSON, E. (org.) Stock Market Anomalies, Cambridge University Press, 1988. 
que os preços das ações seguiam o modelo random walk multiplicativo, ou seja:

$$
P_{t}=P_{t-1}\left\{e^{\left[E\left(R_{t}\right)+e_{t}\right]}\right\}-D I V_{t}
$$

onde:

$P_{t}$ e $P_{t-1}$ são os preços no início e fim do período $t$; DIV $_{t}$ são os dividendos pagos durante o período $t$; $\mathbf{E}\left(\mathbf{R}_{t}\right)$ é o retorno esperado no período t;

$\mathbf{e}_{t}$ é o erro aleatório.

Tomando-se o logaritmo em ambos os lados da equação (2), tem-se:

$$
R_{t}=L_{n}\left[\left(P_{t}+D I V_{t}\right) / P_{t-1}\right]=E\left(R_{t}\right)+e_{t}
$$

Assim, os retornos mensais foram calculados pela seguinte equação:

$$
R_{t}=L_{n}\left(\text { IBOVESPA }_{t} / \text { IBOVESPA }_{t-1}\right)
$$

onde:

$\mathbf{R}_{\mathbf{t}}$ é o retorno do IBOVESPA no mês t;

IBOVESPA $_{t}$ é a cotação de fechamento no último dia do mês $t$, incluindo os dividendos e outras ocorrências das ações que o compõem;

IBOVESPA $_{\mathrm{t}-1}$ é a cotação de fechamento no último dia do mês t-1.

\section{Testes paramétricos}

Para testar a existência de sazonalidade/ anomalia na série de retornos mensais, foram efetuadas duas regressões múltiplas com variáveis mudas.

A primeira regressão, equação (5), serve para testar a hipótese de que os retornos médios mensais são iguais a zero para cada mês do ano $\left(H_{0}: a_{1}=a_{2}=\ldots=a_{12}=0\right)$, fornecendo, portanto, a estimativa dos retornos médios mensais durante o período estudado e a sua significância através da estatística t.

$$
R_{t}=a_{1} D_{1 t}+a_{2} D_{2 t}+\ldots+a_{12} D_{12 t}+e_{t}
$$

onde:

$\mathbf{R}_{\mathbf{t}}$ é o retorno mensal do índice no mês t; $\mathbf{D}_{\text {it }}$ são as variáveis mudas, assim, por exemplo, no mês de janeiro $D_{1 t}=\mathbf{1}$ e $D_{\mathbf{2 t}}=D_{3 t}=\ldots=D_{12 t}=0$;

$\mathbf{a}_{1} \ldots \mathbf{a}_{12}$ são os retornos mensais esperados;

$e_{t}$ é o erro aleatório.

A equação (6) permite testar a hipótese, através da estatística $F$, de que as diferenças entre os retornos dos outros meses e o mês de janeiro são zero $\left(H_{0}: a_{2}=a_{3}=\ldots=a_{12}=0\right)$, o que na verdade, equivale à hipótese de que os retornos médios são iguais para todos os meses do ano $\left(\mathbf{H}_{0}: \mathbf{a}_{\mathbf{1}}=\mathbf{a}_{\mathbf{2}}=\ldots=\mathbf{a}_{12}\right)$.

$$
R_{t}=a_{1}+a_{2} D_{2 t}+\ldots+a_{12} D_{12 t}+e_{t}
$$

onde:

$a_{1}$ é o retorno médio do mês de janeiro;

$\mathbf{a}_{2} \ldots \mathbf{a}_{12}$ são as diferenças entre os retornos dos outros meses e o mês de janeiro;

$D_{i t}$ e $e_{t}$ têm o mesmo significado que na equação (5).

O uso de regressões com variáveis mudas, como nas equações (5) e (6), é adotado na maioria da literatura a respeito de anomalias/sazonalidades do mercado financeiro.

Os resultados obtidos, usando-se as equações acima, estão sumarizados na tabela 1 .

\section{Testes não-paramétricos}

Os testes anteriores têm como pressuposto que a distribuição dos retornos do IBOVESPA é normal.

Outra metodologia é usar testes não-paramétricos. Dessa maneira, não é necessário assumir nenhuma hipótese quanto ao tipo de distribuição a ser testada. Para tanto, foi usado o teste de Kruskal-Wallis ${ }^{16}$ que se baseia no ranking de cada observação para testar se duas ou mais amostras pertencem à mesma população. A única restrição é que as amostras devem ser independentes.

Se a hipótese nula for verdadeira, ou seja, se o retorno mensal for iqual para todos os meses do ano, a estatística de Kruskal-Wallis terá uma distribuição chi-quadrado, aproximadamente, com k-1 graus de liberdade, onde k é o número de amostras testadas.

A tabela 1 mostra o resultado obtido para as duas séries em apreço.

\section{O EFEITO DIA-DA-SEMANA}

Para detectar o efeito dia-da-semana, foram usadas as cotações diárias do IBOVESPA durante o período de janeiro/86 a março/89. Nesse caso a série não foi deflacionada, pois não existe nenhum índice que meça a inflação diária. Aqui, convém observar que, caso fosse determinada uma taxa diária a partir de uma taxa mensal de inflação (usando-se matemática financeira), a primeira seria constante ao longo dos dias do mês e o deflacionamento da série diária não traria nenhuma informação adicional pois, como o objetivo é a comparação entre retornos diários, a divisão de uma série diária por uma constante não vai alterar a posição relativa entre os retornos, a não ser quando parte de uma semana estiver no final de um mês e a outra parte estiver no mês seguinte.

Os retornos foram calculados usando-se a mesma metodologia empregada no cálculo dos retornos mensais; vide equação (4).

16. CONOVER, W.J. Practical Nonparametric Statistics. New York Wiley, 1980. 
Tabela 1:

\section{Estatísticas da distribuiçãa mensal dos retornos do IBOVESPA năo deflacionado}

\begin{tabular}{|c|c|c|c|c|c|c|}
\hline & OBS. & MÉDIA & $\mathbf{t}$ & $\begin{array}{c}\text { DESV. } \\
\text { PADRÄO }\end{array}$ & KURTOSE & ASSIMETRIA \\
\hline JAN & 20 & 0,0934 & $2,74^{\star}$ & 0,1609 & $-0,1113$ & $-0,0051$ \\
\hline FEV & 20 & 0,0536 & 1,57 & 0,0887 & $-0,8383$ & 0,1070 \\
\hline MAR & 20 & 0,0796 & $2,34^{* *}$ & 0,2032 & $2,4485^{* *}$ & $1,8101^{*}$ \\
\hline ABR & 20 & 0,0773 & $2,27^{\star *}$ & 0,1430 & $-0,7630$ & 0,0464 \\
\hline MAI & 20 & 0,0966 & $2,84^{*}$ & 0,1507 & $-0,3633$ & 0,5581 \\
\hline JUN & 20 & 0,0560 & 1,64 & 0,1561 & $-0,1990$ & 0,8850 \\
\hline JUL & 20 & 0,0491 & 1,44 & 0,1033 & $-0,3617$ & 0,2723 \\
\hline AGO & 20 & 0,0444 & 1,31 & 0,1398 & $-0,9935$ & -0.1780 \\
\hline SET & 20 & 0,0441 & 1,30 & 0,1693 & $-0,9451$ & 0,2578 \\
\hline OUT & 20 & 0,0351 & 1,03 & 0,1690 & $-1,3264$ & 0,3720 \\
\hline NOV & 20 & 0,0285 & 0,84 & 0,1573 & $-0,2856$ & 0,5460 \\
\hline DEZ & 20 & 0,0553 & 1,63 & 0,1513 & $-0,0801$ & 0,8562 \\
\hline
\end{tabular}

$\mathbf{F}=0,43$

$H=6,21$ (estat. de Kruskal-Wallis)

\section{Estatísticas da distribuição mensal dos retornos do IBOVESPA deflacionado}

\begin{tabular}{|c|c|c|c|c|c|c|}
\hline & OBS. & MÉDIA & 1 & $\begin{array}{l}\text { DESV. } \\
\text { PADRÄ́O }\end{array}$ & KURTOSE & ASSIMETRIA \\
\hline JAN & 20 & 0,0336 & 1,06 & 0,1618 & $-0,0240$ & $-0,6341$ \\
\hline FEV & 20 & $-0,0047$ & -0.15 & 0.0970 & 0,1363 & $-0,3709$ \\
\hline MAR & 20 & 0,0208 & 0.66 & 0.1932 & $2,1704^{* *}$ & $1.5996^{*}$ \\
\hline $\mathrm{ABR}$ & 20 & 0,0254 & 0,80 & 0.1310 & $-0,3449$ & $-0,0311$ \\
\hline MAI & 20 & 0,0428 & 1.35 & 0,1668 & 0,4929 & 0,0933 \\
\hline JUN & 20 & $-0,0003$ & $-0,01$ & 0.1297 & 0,4903 & $1,0840^{\star *}$ \\
\hline JUL & 20 & $-0,0023$ & $-0,07$ & 0.1170 & -0.8752 & 0.2144 \\
\hline AGO & 20 & $-0,0068$ & $-0,22$ & 0,1184 & $-0,9136$ & $-0,2956$ \\
\hline SET & 20 & $-0,0075$ & -0.24 & 0.1354 & $-1,2189$ & $-0,1192$ \\
\hline OUT & 20 & $-0,0197$ & $-0,62$ & 0,1440 & $-0,9404$ & 0.0010 \\
\hline MOV & 20 & $-0,0269$ & $-0,85$ & 0,1435 & $-0,3840$ & 0.6031 \\
\hline DEZ & 20 & $-0,0015$ & $-0,05$ & 0,1389 & $-0,7886$ & 0,1832 \\
\hline \multicolumn{7}{|c|}{$\begin{array}{l}F=0,46 \\
H=6,73 \text { (estat. de Kruskal-Wallis) }\end{array}$} \\
\hline
\end{tabular}

\section{Testes Paramétricos}

Para calcular os retornos diários e testar a hipótese de igualdade dos retornos nos cinco dias da semana, foram usadas equações semelhantes às equações (5) e (6), da seção anterior.

$$
R_{t}=a_{1} D_{1 t}+a_{2} D_{2 t}+a_{3} D_{3 t}+a_{4} D_{4 t}+a_{5} D_{5 t}+e_{t}(7)
$$

onde:

$\mathbf{R}_{\mathbf{t}}$ é o retorno diário do índice no dia t;
$\mathbf{D}_{\mathrm{it}}$ são as variáveis mudas, indicam o dia da semana em que foi observado o retorno, assim, para segunda-feira $D_{1 t}=1$ e $D_{2 t}=\ldots=D_{5 t}=0$;

$\mathbf{a}_{1} \ldots \mathbf{a}_{5}$ são os retornos diários esperados;

$e_{t}$ é o erro aleatório.

A outra regressão é dada pela seguinte equação:

$R_{t}=a_{1}+a_{2} D_{2 t}+a_{3} D_{3 t}+a_{4} D_{4 t}+a_{5} D_{5 t}+e_{t}$ 
onde:

$a_{1}$ é o retorno na $2^{\mathrm{a}}$ feira;

$a_{2} \ldots a_{5}$ são as diferenças entre os retornos dos outros dias da semana e $2^{\underline{a}}$ feira; (7).

$D_{\text {it }}$ e $e_{t}$ têm o mesmo significado que na equação

Caso os retornos sejam os mesmos para cada um dos dias da semana, as estimativas de $\mathbf{a}_{2}$ até $\mathbf{a}_{5}$, na equação (8), não serão significativamente diferentes de zero e a estatística F que mede a significância conjunta dessas variáveis não deverá ser significante. Os resultados referentes às estatísticas das distribuições dos retornos mensais são apresentados na tabela 2 .

\section{Testes Não-Paramétricos}

Da mesma maneira que os retornos mensais, para os retornos diários foi aplicado o teste de KruskalWallis que não presupõe nenhum tipo de distribuição na população a ser testada, apenas a independência das amostras. Esse resultado é apresentado na tabela 2 .

\section{CONCLUSÕES}

Os resultados obtidos na tabela 1 mostram que, no período estudado, não foi detectado nenhum efeito mês-do-ano, pois o valor de $\mathrm{F}=0,43$, para a série não deflacionada e $\mathrm{F}=0,46$, para a série deflacionada, não são significativos. Esse resultado, de certa forma, proporciona mais uma contribuição favorável à hipótese do efeito tax-loss selling, pois o mercado de capitais brasileiro não possuía imposto sobre ganhos de capital, na época da coleta de dados. Essa hipótese diz que em mercados onde existe taxação sobre ganhos de capital, os investidores que possuem açōes que sofreram perdas de valor ao longo do ano são motivados a vendê-las no mês de dezembro para fins de abatimento no imposto de renda. No início do próximo exercício, com o fim das pressões artificiais de venda, o mercado volta ao equilíbrio e, portanto, as ações que tiveram um declínio artificial no final do período anterior sofrem um retorno acima do normalmente esperado.

Quanto ao efeito dia-da-semana, pelos resultados da tabela 2, percebe-se que é significativo, a nível de $1 \%$. O efeito observado é semelhante ao do mercado americano, onde o menor retorno é na segunda-feira (negativo) e o maior, na sexta-feira. Essa anomalia também é denominada efeito fim-de-semana. Até agora, não existe nenhuma explicação razoável para o efeito dia-da-semana ou efeito fim-de-semana. Alguns autores atribuem esse efeito às regras de liquidação do mercado (por exemplo, na Bovespa a liquidação física é feita em três dias e a financeira em cinco) e aos "juros extras" durante o final de semana ${ }^{17}$. Já Penman ${ }^{18}$ parte para uma argumentação mais para o lado da psicologia e propõe como hipótese causadora do efeito fim-de-semana a de que as empresas tendem a tornar públicas más notícias somente depois que o mercado fechou na sextafeira, de maneira que os investidores tenham mais tempo em absorvê-las.

A conclusão que se pode tirar dessas e de uma série de outras anomalias/sazonalidades detectadas em diferentes tipos de mercados de diferentes países é que, realmente, essas anomalias existem e cabe agora determinar as causas subjacentes a elas, ou então, é a própria noção de mercado eficiente que precisa ser redefinida e/ou os modelos de equilíbrio de ativos de risco (CAPM, APT etc.) precisam incorporar novas variáveis explicativas para essas diversas anomalias $^{19} . \square$

17. LAKONISHOK, J. \& LEVI, M. Op. cit.

18. PENMAN, S.H. "The Distribution of Earnings News Over Time and Seasonalities in Aggregate Stock Returns". Journal of Financial Economics, 18:199-228, 1987.

19. Gostaria de agradecer a Jonh O'Hanlon, do Departamento de Contabilidade e Finanças, da Universidade de Lancaster, por suas críticas e sugestões. No entanto, qualquer erro ou omissão é de minha inteira responsabilidade.

Tabela 2: Estatísticas da distribuição diária dos relornos do IBOVESPA

\begin{tabular}{|c|c|c|c|c|c|c|}
\hline & OBS. & MÉDIA & $t$ & $\begin{array}{l}\text { DESV. } \\
\text { PADRÃO }\end{array}$ & KURTOSE & ASSIMETRIA \\
\hline $2^{a}$ & 139 & $-0,0068$ & $-2,33^{* *}$ & 0,0399 & $-0,6420$ & $-0,0860$ \\
\hline $3^{\mathbf{a}}$ & 146 & 0,0023 & 0,82 & 0,0324 & $-0,4299$ & $-0,1069$ \\
\hline $4^{a}$ & 157 & 0,0121 & $4,40^{*}$ & 0,0329 & 0,1361 & 0,1167 \\
\hline $5^{\mathbf{a}}$ & 153 & 0,0063 & $2,26^{* *}$ & 0,0351 & 0,5346 & $-0,0346$ \\
\hline $6^{a}$ & 155 & 0,0127 & $4,60^{*}$ & 0,0315 & $-0,6607$ & $-0,1224$ \\
\hline \multicolumn{7}{|c|}{$\begin{array}{l}\mathbf{F}=7,90^{*} \\
\mathbf{H}=27,12^{\star} \text { (estat. de Kruskal-Wallis) }\end{array}$} \\
\hline \multicolumn{7}{|c|}{$\begin{array}{l}* \quad \text { significante ao nivel de } 1 \% \\
* \text { * } \text { significante ao nivel de } 5 \%\end{array}$} \\
\hline
\end{tabular}

